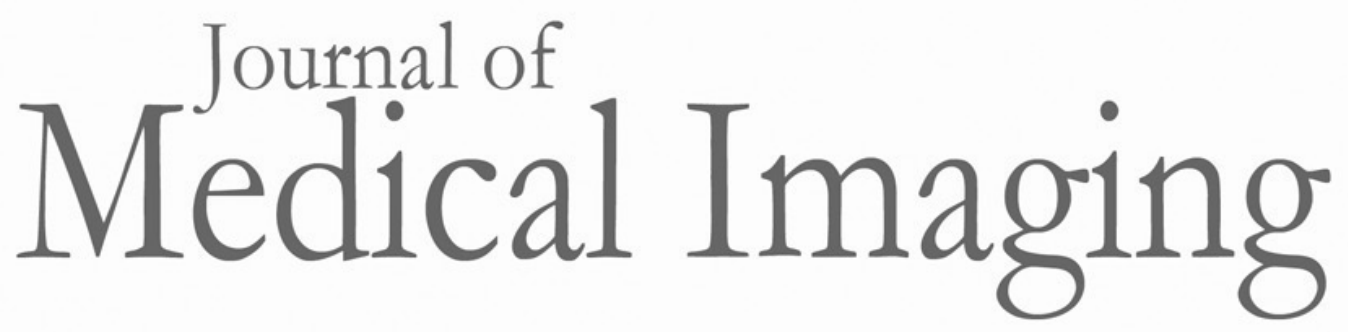

Medicallmaging.SPIEDigitalLibrary.org

\title{
2019 List of Reviewers
}


The Journal of Medical Imaging would like to sincerely thank the following individuals who served as reviewers in 2019. The success of our publication hinges on the voluntary contributions of time and energy put forth by these professionals.

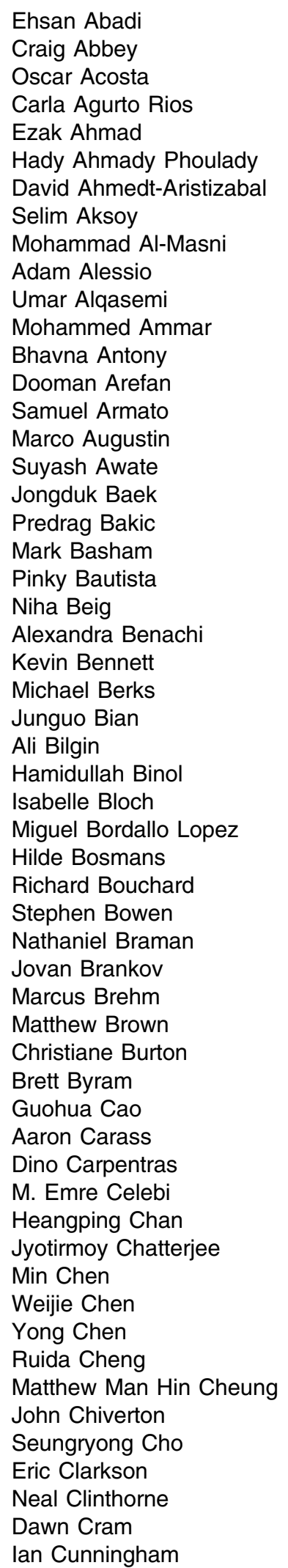

\author{
Mehmet Dalmis \\ Hao Dang \\ Bruno De Man \\ Tharindu De Silva \\ Bob de Vos \\ Oliver Diaz \\ Olaf Dietrich \\ Alexey Dimov \\ Leila Djerou \\ Lei Dong \\ James Dormer \\ Qi Dou \\ Trafton Drew \\ Karen Drukker \\ Alisha DSouza \\ James Duncan \\ Madeleine Durkee \\ Rudresh Dwivedi \\ Brendan Eck \\ Anders Eklund \\ Hany Elsalamony \\ Bradley Erickson \\ Rebecca Fahrig \\ Yong Fan \\ Baowei Fei \\ Farzad Fereidouni \\ Sean Foxley \\ Joseph Foy \\ Winnie $\mathrm{Fu}$ \\ Olac Fuentes \\ Jordan Fuhrman \\ Brandon Gallas \\ Nagarajan Ganapathy \\ Grace Gang \\ Aimilia Gastounioti \\ Marios Gavrielides \\ Arkadiusz Gertych \\ Zahra Ghanian \\ Ardalan Ghasemzadeh \\ Peyman Gholami \\ Luca Giannoni \\ Howard Gifford \\ Randolph Glickman \\ Evgin Goceri \\ Hao Gong \\ Marlies Goorden \\ Mohammed Goryawala \\ Alexej Gossmann \\ William Grissom \\ Huifeng Guan \\ Shuyue Guan \\ Eyjolfur Gudmundsson \\ Cigdem Gunduz-Demir \\ Metin Gurcan \\ Tim Gureyev \\ Lubomir Hadjiiski
}

Lawrence Hall

Wenchao Han

Steven Hart

Douglas Hartman

Adam Hasse

Yuji Hatanaka

Mathieu Hatt

Daniel Hausermann

Tamara Haygood

Phengann Heng

Goretti Hernandez Cardoso

Karl-Heinz Herrmann

William E. Higgins

Stephen Hillis

Yasushi Hirano

Kenneth Hoffmann

Todd Horowitz

Hasan Hosseinzadeh

Carrie Hruska

Scott Hsieh

Henkjan Huisman

Andreas Husch

Hayato Itoh

Oleksandra Ivashchenko

Jayender Jagadeesan

Andrew Janowczyk

Uditha Jayarathne

Yong Jeong

Anna Jerebko

Abhinav Jha

Songbai Ji

Wei Ji

Jun Jiang

Yueming Jin

Shiju Joseph

Marc Kachelriess

Le Kang

Anuj Kapadia

Steffen Kappler

Andrew Karellas

Karim Karim

Melinda Katona

Hiroshi Kawaguchi

Yoshiki Kawata

Michal Kawulok

Sungsoo Kim

Thomas Kirchner

Felipe Kitamura

Takayuki Kitasaka

Ellen Kok

Cemal Kose

Kivanc Kose

Ganapathy Krishnamurthi

Elizabeth Krupinski

Hugo Kuijf

Naveen Kumar 


\begin{tabular}{|c|c|c|}
\hline Asli Kumcu & Giacomo Nebbia & Caifeng Shan \\
\hline Hsienchi Kuo & Hamid Neshat & Gregory Sharp \\
\hline Kemi Ladeji-Osias & Muhammad Khalid Khan & Jennifer Shell \\
\hline Hariharan Lalgudi & Niazi & Bibo Shi \\
\hline Bennett Landman & Robert Nishikawa & Jie Shi \\
\hline Frederic Lange & Tianye Niu & Pavel Shilyagin \\
\hline Patrick LaRiviere & Jack Noble & Emil Sidky \\
\hline Guenter Lauritsch & Peter Noel & Milan Sinka \\
\hline Charles Laymon & Jose Noguera & Teri Sippel Schmidt \\
\hline Silas Leavesley & Ioan Notingher & Arkadiusz Sitek \\
\hline Christoph Lee & Christine O'Brien & Justin Solomon \\
\hline Jinseok Lee & Hirohisa Oda & Chaolong Song \\
\hline Juhun Lee & Tim Olson & Jacob Sosna \\
\hline Patrick Leo & Adelaja Osibote & Aristeidis Sotiras \\
\hline Nikolas Lessmann & David Paganin & Steven Squires \\
\hline Samuel Leung & Santosh Paidi & Marius Staring \\
\hline Chiye Li & Tinsu Pan & J. Webster Stayman \\
\hline Feng Li & Shuo Pang & Darko Stern \\
\hline $\mathrm{Li} \mathrm{Li}$ & Deepa Parasar & Lin Su \\
\hline Peng Li & Min-Chul Park & Takaaki Sugino \\
\hline Weiguo Li & Andres Parra & Mark Supanich \\
\hline Weizhe Li & Hari Paudel & Hidenobu Suzuki \\
\hline Yusheng Li & Yannis Paulus & Timothy Szczykutowicz \\
\hline Wenxuan Liang & Raquel Perez Lopez & Jianbo Tang \\
\hline Gilbert Lim & Mats Persson & Xiangyang Tang \\
\hline Cristian Linte & Ricardo Pizarro & Jesse Tanguay \\
\hline Changgeng Liu & William Plishker & Hannah Thomas \\
\hline Haixia Liu & Munish Puri & Anders Tingberg \\
\hline Shuang Liu & Xin Qi & Pallavi Tiwari \\
\hline Xinyang Liu & Kumar Rahul & Junichi Tokuda \\
\hline Yun Liu & Karunanithi Rajamanickam & John Tomaszewski \\
\hline Clara Llorens-Quintana & Luis Isaac Ramos Garcia & Turid Torheim \\
\hline Murray Loew & Gabriel Ramos-Llorden & Benjamin Tsui \\
\hline Renata Longo & Andrik Rampun & James Uhlir \\
\hline Cheng Lu & Robert Raphael & Tamas Ungi \\
\hline Yihuan Lu & Essam Rashed & Martin Urschler \\
\hline Shouhua Luo & Thomas Rau & Sebastien Valette \\
\hline Xiongbiao Luo & Ingrid Reiser & Marijn van Stralen \\
\hline Chi Ma & Samuel Remedios & Srinivasan Vedantham \\
\hline Dan Ma & Liqiang Ren & Dmitry Vengertsev \\
\hline Calum MacAulay & Michael Richards & Francis Verdun \\
\hline Alistair Mackenzie & Taylor Richards & Nishant Verma \\
\hline Ananth Madhuranthakam & Luigi Rigon & Satish Viswanath \\
\hline S. Mahdavi & Georg Rose & Justin Wan \\
\hline James Mainprize & Sean Rose & Chenglong Wang \\
\hline Awais Mansoor & Theo Ruers & Hesheng Wang \\
\hline Oge Marques & Sudipan Saha & Jinghui Wang \\
\hline Douglas Mast & Firdous Saleheen & Shouyu Wang \\
\hline Diana Mateus & Ravi Samala & Juergen Weese \\
\hline Zahid Mehmood & Frank Samuelson & Dong Wei \\
\hline Carlos Mello & Jeremiah Sanders & Simon Weidert \\
\hline Claudia Mello-Thoms & Pinaki Sarder & Andreas Weinmann \\
\hline Fausto Milletari & Helmut Schlattl & Si Wen \\
\hline Dimple Modgil & Ioannis Sechopoulos & Heather Whitney \\
\hline James Monaco & William Paul Segars & Arnold Wiliem \\
\hline Stephen Moore & Anando Sen & Lauren Williams \\
\hline Mehdi Moradi & Baha Sen & Eric Wolsztynski \\
\hline Amin Motahari & Randolph Setser & Dufan Wu \\
\hline Ethan Murphy & Lingdao Sha & Jia Wu \\
\hline Peter Naglic & Muhammad Shafiq ul Hassan & Adam Wunderlich \\
\hline Sandy Napel & Amir Shahmoradi & Yijing Xie \\
\hline
\end{tabular}


2019 List of Reviewers

Zhenyu Xiong

Martin Yaffe

Guang Yang

Jiarui Yang

Robail Yasrab

Xin Yin

Jie Yuan

Yading Yuan

Fatemeh Zabihollahy
Simon Zabler

Sahar Zafari

Haishan Zeng

Ke Zeng

Jie Zhang

Jing Zhang

Lei Zhang

Lei Zhang

Ruoqiao Zhang
Shengjun Zhang

Lei Zhao

Wei Zhao

Wei Zhao

Yanyu Zhao

Yiyuan Zhao

Wei Zhou 